\title{
A tale of two LAMS: a report of benign tissue ingrowth resulting in recurrent gastric outlet obstruction
}

\section{다)(1) $\odot$}

Authors

Parth J. Parekh, Mohammad H. Shakhatreh, Paul Yeaton

Institution

Department of Internal Medicine, Division of

Gastroenterology, Carilion Clinic, Roanoke, Virginia,

United States

submitted 5.1.2018

accepted after revision 25.6.2018

Bibliography

DOI https://doi.org/10.1055/a-0658-1211 |

Endoscopy International Open 2018; 06: E1390-E1394

(c) Georg Thieme Verlag KG Stuttgart · New York

ISSN 2364-3722

Corresponding author

Parth J. Parekh, MD, Department of Internal Medicine, Division of Gastroenterology, Carilion Clinic, Roanoke,

VA 24016

Fax: +1-504-981-7005

parthjparekh@gmail.com

\begin{abstract}
Background and study aims Endoscopic ultrasound (EUS)-guided gastroenterostomy is a novel, off-label, procedure that has been facilitated by availability of a novel lumen apposing metal stent (LAMS) (Axios, Boston Scientific, Marlbourough Massachusetts, United States). The literature suggests its efficacy to be comparable to surgical gastrojejunostomy. In theory, this procedure overcomes the issues associated with woven enteral stents, which are subject to continuous, straightening, axial forces. We report the adverse event of tissue ingrowth in an Axios stent placed as an EUS-guided gastroduodenostomy, resulting in recurrence of gastric outlet obstruction, treated by a stentinside-stent technique.
\end{abstract}

\section{Introduction}

Pancreatic adenocarcinoma may be complicated by gastric outlet obstruction, resulting in abdominal pain, nausea, and vomiting. Traditional treatment options include surgical bypass and endoscopic enteral stent placement. The available enteral stents are all woven; their high axial forces [1] result in a persistent straightening force which predisposes the devices to failure. Surgical gastrojejunostomy is relatively invasive, particularly in patients with advanced malignancies and poor nutritional status. Endoscopic ultrasound-guided gastroenterostomy (EUS-GE) is a promising technique, as studies [2] have demonstrated that it overcomes the shortcomings of both enteral stent placement and surgical intervention. We report a case of benign tissue ingrowth complicating placement of an EUS-guided gastrojejunostomy Axios stent placed in the setting of malignant gastric outlet obstruction. This was successfully managed by placing a second Axios stent inside the first, resulting in tissue necrosis of the ingrowth in the first stent, following which both stents were removed.

\section{Case report}

An 86-year-old male with a history of locally advanced, unresectable pancreatic adenocarcinoma presented with recurrent symptoms of gastric outlet obstruction.

His original diagnosis was made in August 2014; prior treatment included biliary stenting (August 2014), chemotherapy (treated with gemcitabine only to develop gemcitabine-induced thrombotic thrombocytopenic purpura a year later, in mid-2015, forcing discontinuation of therapy). In May 2016, he presented with epigastric pain, nausea, and vomiting with cross-sectional imaging demonstrating no significant change in pancreatic mass size and intact stents with stable pneumobilia.

An esophagogastroduodonoscopy (EGD) was performed, demonstrating a narrowing of the second portion of the duodenum that could not be traversed by the endoscope. Contrast medium injected into the bulb showed narrowing of the second portion, measuring approximately $3 \mathrm{~cm}$, consistent with a malignant gastric outlet obstruction (GOO). A Boston-Scientific (Marlborough, Massachusetts, United States) electrocautery- 

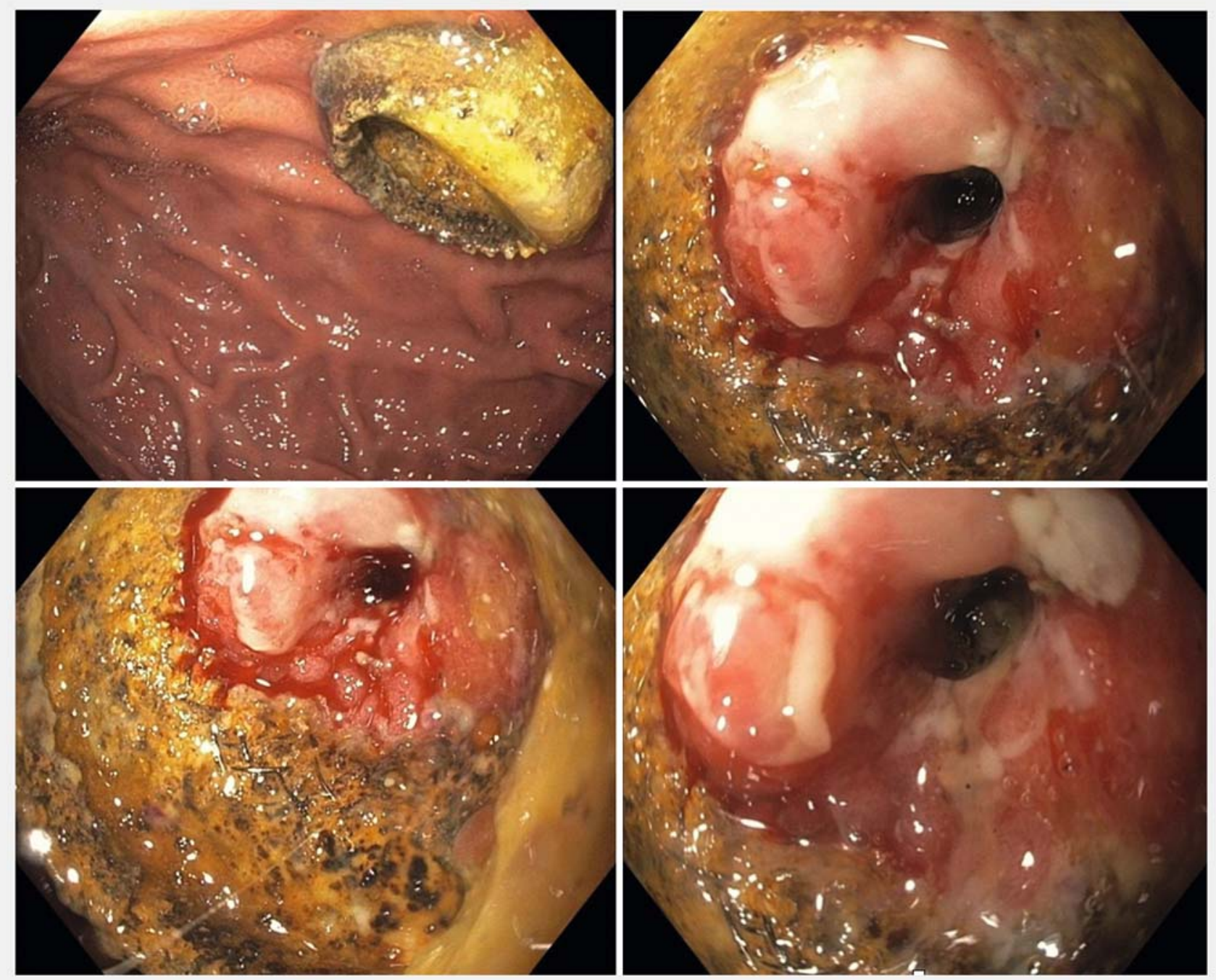

- Fig. 1 Previously placed Axios stent.

enhanced $15 \mathrm{~mm} \times 10 \mathrm{~mm}$ Axios stent was then successfully deployed, under EUS guidance, bypassing the malignant GOO. Shortly after placement of his Axios, the patient returned with right-upper quadrant pain and fever. A novel approach to endoscopic retrograde cholangiopancreatography through a gastrojejunal LAMS was then performed, which further described in detail elsewhere [3]. In the next 6 months, the patient had two such repeat procedures for recurrent cholangitis due to an occluded fully-covered metal biliary stent, both of which demonstrated a patent LAMS.

In July 2017, the patient was admitted with complaints of abdominal pain, nausea, and vomiting. He was hemodynamically stable, with subsequent evaluation excluding infection or biliary obstruction. Computed tomography demonstrated pneumobilia and gastric distension with gas and fluid, raising the possibility of recurrent gastric outlet obstruction. Upper endoscopy was performed which demonstrated failure of the stent covering with circumferential tissue ingrowth nearly obliterating the gastrojejunostomy lumen ( $\bullet$ Fig. 1 ).
A biliary retrieval balloon preloaded with a guidewire was advanced into the jejunum and a second LAMS deployed within the first LAMS. A 10- to $13-\mathrm{mm}$ dilation balloon was centered in the stent and inflated to $13 \mathrm{~mm}$ ( $\vee$ Fig. 2) with fluoroscopic control. The endoscope was then reintroduced and advanced into the lumen of the LAMS, where normal-appearing small bowel mucosa was observed ( $\triangleright$ Fig.3). The patient had immediate symptom resolution and was discharged, demonstrating ability to tolerate a regular diet.

In August 2017, the patient returned to our endoscopy suite for removal of the LAMS. Using a snare, the inner LAMS was removed successfully, exposing the initial stent. Persistent tissue ingrowth was observed. The second LAMS was removed successfully using a snare and a new LAMS successfully deployed into the fistulous tract ( $\vee$ Fig. 4 ). The patient was seen in clinic 1 month later and is currently tolerating an unrestricted diet without difficulty. 

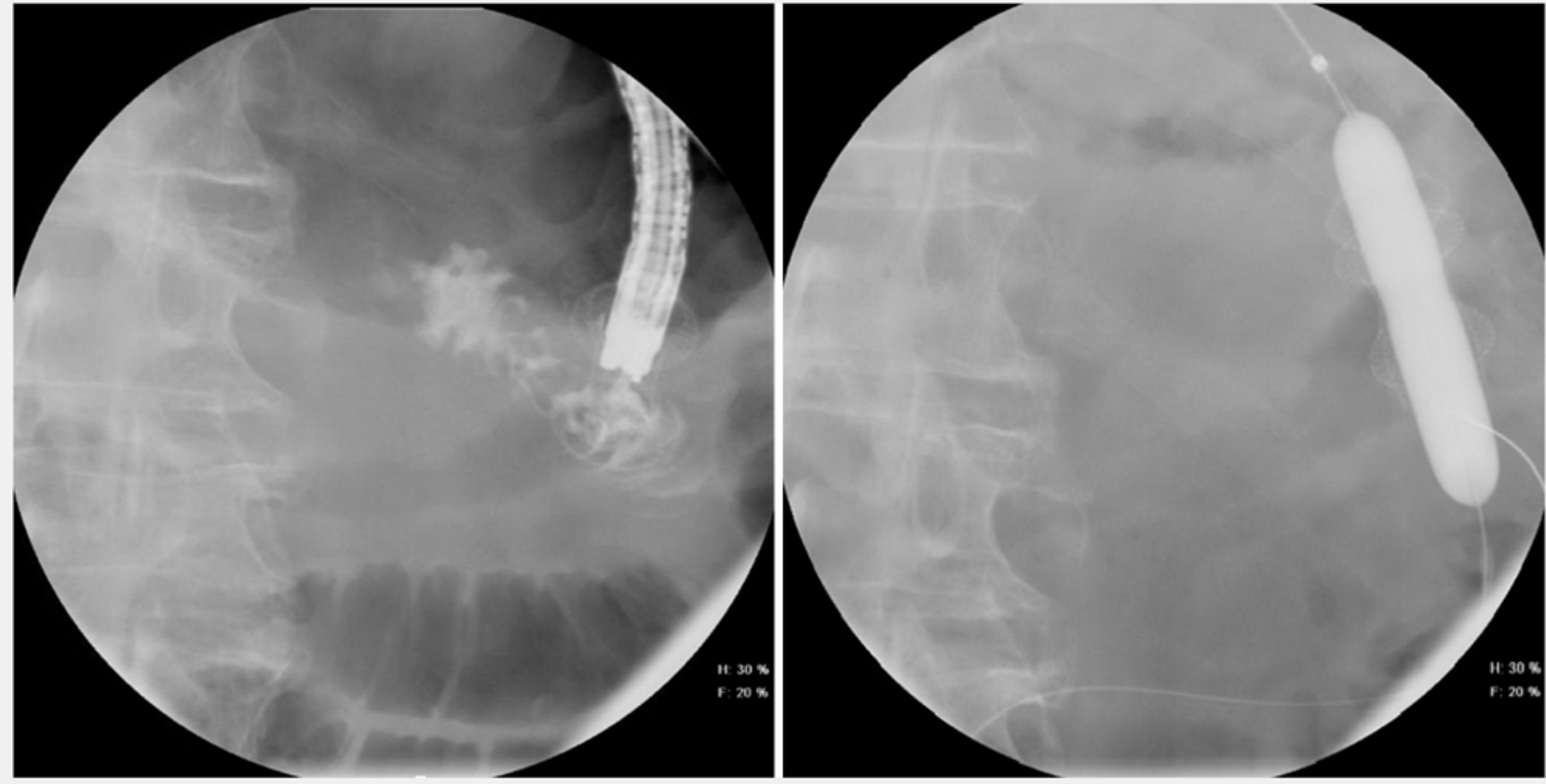

Fig. 2 Balloon dilation.
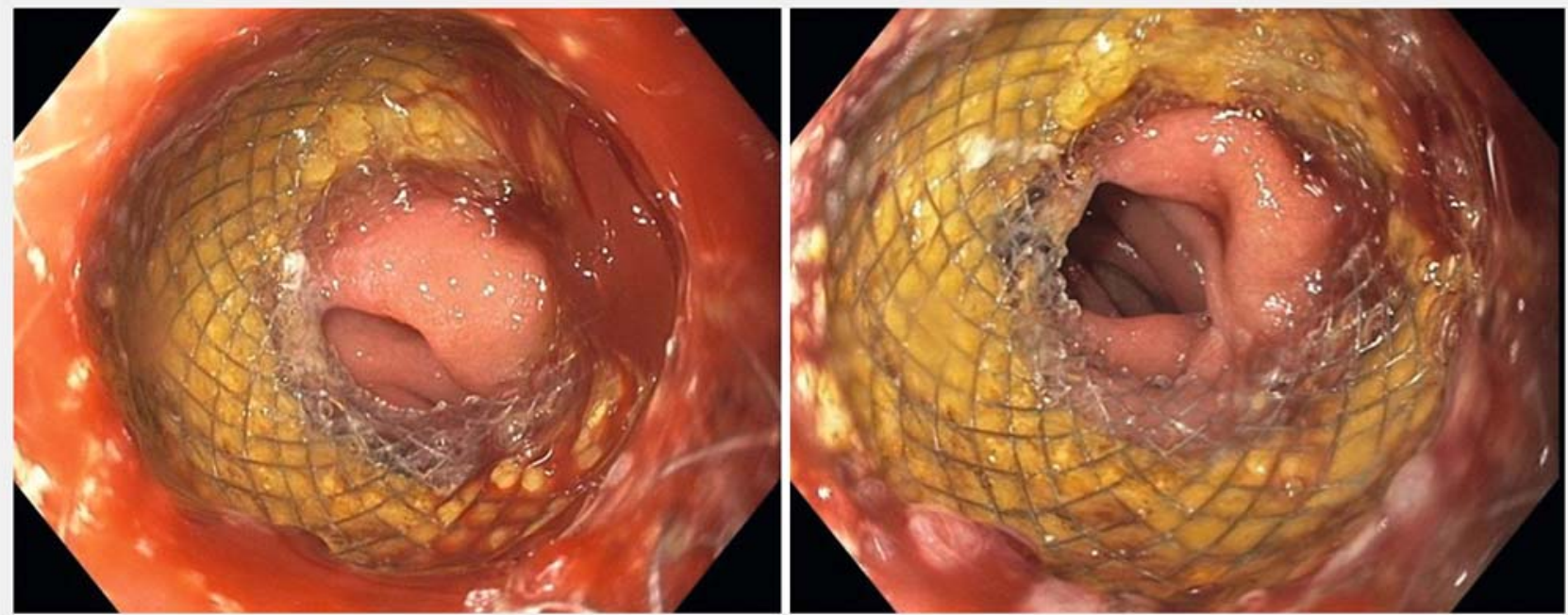

- Fig. 3 Post-Axios stent deployment and balloon dilation.

\section{Discussion}

GOO is a clinical syndrome characterized by epigastric abdominal pain, nausea, vomiting, and inability to tolerate oral intake. Malignant GOO occurs in the setting of a variety of cancers, including pancreatic adenocarcinoma. Current estimates suggest that upwards of $25 \%$ of patients with pancreatic cancer will develop GOO [2]. The ultimate goal of therapy in this difficult-totreat population is to improve quality of life and maintain adequate nutritional status.
Traditional palliative treatment options include surgical gastrojejunostomy to bypass the obstruction or endoscopic placement of a duodenal enteral stent directly across the obstruction. Data comparing the two remain limited but suggest that patients who have endoscopic enteral stent placement experience faster relief of symptoms when compared to surgery. Symptom relief may prove transient compared with surgical bypass, as enteral stents reportedly are associated with more adverse events, recurrence of symptoms over the long-term, and more frequent need for intervention [4-7]. Presumably this is due to the design of the available devices, all of which are 

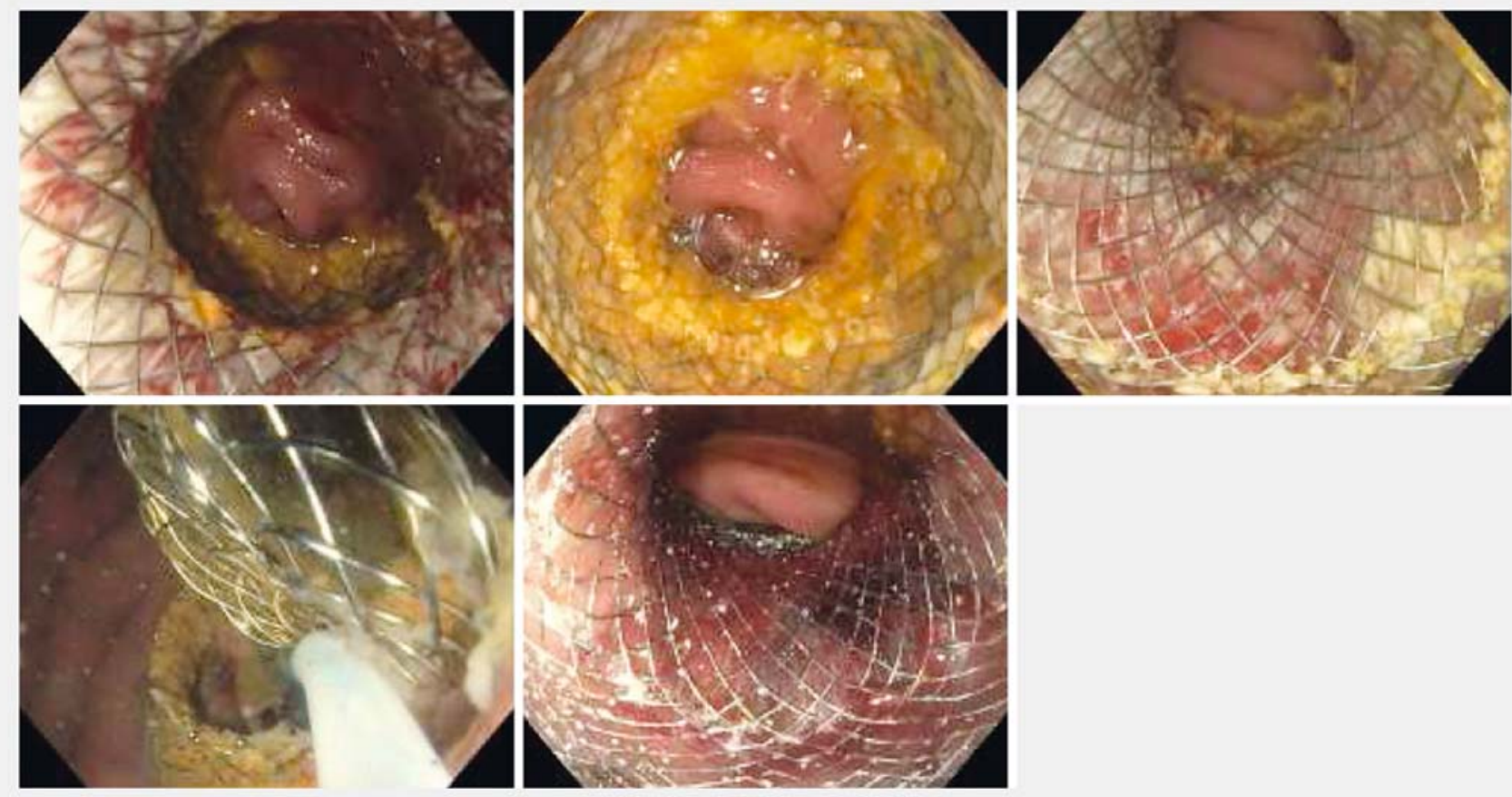

Fig. 4 Removal of previously placed axios stents and post-axios stent deployment of a new axios.

formed of a woven metal platform. Such devices possess varying degrees of axial force [1], exerting a continuous force as the stent attempts to become straight. EUS-GE has emerged as a promising technique for management of malignant GOO, exploiting the physical characteristics of the Axios stent in an off-label indication. This is a short, woven device with a shape that entraps expanded tissues, resulting in serosal adhesion. The stent is fully covered with a proprietary silicone-based material, intended to prevent tissue ingrowth and facilitate removal.

A recent international retrospective study compared the clinical and technical success, adverse events, length of hospital stay, and symptom recurrence in 93 patients treated with either EUS-GE $(n=30)$ or surgical gastrojejunostomy $(n=63)$ [8]. Despite a higher technical success rate in the surgical cohort, the clinical success rate was equal between the two groups. In addition, the rate of adverse events was lower in the EUS-GE cohort, although not statistically significant. Hospital stay and mean time to reintervention were similar; EUS-GE was found to be equally effective and safe compared to surgical gastrojejunostomy. There were no reported cases of tissue ingrowth in this study. Similar studies have also demonstrated long-lasting symptom palliation of $\mathrm{GOO}$ similar to that of surgical bypass, in part owing to low risk of tissue ingrowth $[8,9]$.

\section{Conclusion}

We report benign tissue ingrowth of an Axios stent in the setting of malignant GOO, successfully treated by a second Axios placed within the previously placed stent. It remains unclear if the larger-diameter Axios, which recently became available in the United States, would mitigate this adverse event. Alteration of the covering could also prevent future reports of tissue ingrowth.

\section{Competing interests}

None

\section{References}

[1] Isayama H, Nakai Y, Toyokawa Y et al. Measurement of radial and axial forces of biliary self-expandable metallic stents. Gastrointest Endosc 2009; 70: $37-44$

[2] Irani S, Baron TH, Itoi T et al. Endoscopic gastroenterostomy: techniques and review. Curr Opin Gastroenterol 2017; 33: 320 - 329

[3] Shakhatreh MH, Yeaton P. ERCP through a gastrojejunal lumen-apposing stent. VideoGIE 2016; 1: 19-21

[4] Adler DG, Baron TH. Endoscopic palliation of malignant gastric outlet obstruction using self-expanding metal stents: experience in 36 patients. Am J Gastroenterol 2002; 97: 72 - 78

[5] Tringali A, Didden P, Repici A et al. Endoscopic treatment of malignant gastric and duodenal strictures: a prospective, multicenter study. Gastrointest Endosc 2014; 79: 66 - 75 
[6] Jeurnink SM, Steyerberg EW, Van hooft JE et al. Surgical gastrojejunostomy or endoscopic stent placement for the palliation of malignant gastric outlet obstruction (SUSTENT study): a multicenter randomized trial. Gastrointest Endosc 2010; 71: 490 - 499

[7] Jeurnink SM, Polinder S, Steyerberg EW et al. Cost comparison of gastrojejunostomy versus duodenal stent placement for malignant gastric outlet obstruction. J Gastroenterol 2010; 45: 537-543
[8] Khashab MA, Bukhari M, Baron TH et al. International multicenter comparative trial of endoscopic ultrasonography-guided gastroenterostomy versus surgical gastrojejunostomy for the treatment of malignant gastric outlet obstruction. Endosc Int Open 2017; 5: E275-E281

[9] Khashab MA, Baron TH, Binmoeller KF et al. EUS-guided gastroenterostomy: a new promising technique in evolution. Gastrointest Endosc 2015; 81: $1234-1236$ 